advantageous. Recently, plasma membrane calcium ATPase 4 (PMCA) has been established as a novel mediator of angiogenesis through its role in endothelial cell migration and tubule formation. In addition to PMCA4, both PMCA1 and PMCA2 are also expressed in human endothelial cells but their contribution to angiogenesis remains unknown. Therefore, we hypothesise that PMCA1 also modulates angiogenesis by altering endothelial cell behaviours.

Transient knockdown of PMCA1 was achieved in human umbilical vein endothelial cells (HUVECs) using siRNA (siPMCA1) and confirmed with GPCR and western blot. Assays to asses HUVEC viability, proliferation and rate of apoptosis were performed over a period of 6 days post-transfection using Alamar Blue, Ki-67 immunofluorescence and CaspaseGlo 3/7 respectively. In addition, live-cell imaging was performed over a period of 21 hours to further evaluate migration of cells in a wound, cell necrotic cytotoxicity and cell apoptotic death. Furthermore, HUVECs were added to Matrigel to evaluate in-vitro tubule formation and DAPI-stained cells were subject to fluorescent activated cell sorting to determine cell-cycle distribution. Molecular mechanisms were investigated using Western blot.

Transient knockdown of PMCA1 in HUVECs resulted in an $85 \%$ reduction of PMCA1 at both mRNA and protein level but did not alter the levels of PMCA4 mRNA up to 6 days post transfection. Phenotypically, 3 and 6 days posttransfection, loss of PMCA1 significantly reduced HUVEC viability without a concomitant increase in HUVEC apoptosis or cytotoxicity. Immunofluorescent staining for Ki-67 showed no statistical difference between si-PMCA1 and control HUVECs for the percentage of Ki-67 positive cells. However, FACsmediated cell cycle analysis revealed si-PMCA1 HUVECs had a significantly larger proportion of cells in G0/G1 phase with fewer cells identified in G2/M-phase compared to control HUVECs after serum starvation followed by 8 hours of full serum media. Additionally, live-cell imaging of the wound healing assay revealed si-PMCA1 HUVECs displayed significantly reduced HUVEC wound density and wound confluence, resulting in an increased wound width over time. Furthermore, si-PMCA1 also show reduced ability to form tubules invitro characterised by a reduction in the number of tubules and tubule junctions formed, despite si-PMCA1 HUVECs having higher protein levels of the pro-angiogenic gene RCAN1.4.

Overall, transient knockdown of PMCA1 has adverse effects on HUVEC viability, migration and tubule formation suggesting loss of PMCA1 is detrimental for in-vitro angiogenesis.

\section{SENESCENCE AS A THERAPEUTIC TARGET FOR MYOCARDIAL AGEING} ${ }^{1}$ Anna Walaszczyk*, ${ }^{1}$ Emily Dookun, ${ }^{1}$ Rachael Redgrave, ${ }^{1}$ Simon Tual-Chalot,
${ }^{1}$ Rhys Anderson, ${ }^{1}$ loakim Spyridopoulos, ${ }^{1}$ Andrew Owens, ${ }^{1}$ Helen Arthur, ${ }^{2}$ Joao Passos,
${ }^{1}$ Gavin Richardson. 'Institute of Genetic Medicine, Newcastle University; ${ }^{2}$ Institute for
Ageing, Newcastle University

\subsection{6/heartjnl-2018-BCS.105}

Ageing is the biggest risk factor for impaired cardiovascular health, cardiovascular disease being the leading cause of death in $40 \%$ of individuals over 65 years old. Ageing is associated not only with an increased prevalence of cardiovascular disease but also with a poorer prognosis, including increased mortality or incidence of heart failure after myocardial infarction (MI).

We have demonstrated that aged (23 month old) mice have an accumulation of cardiomyocyte senescence, reduced regenerative potential and display increased mortality as well as impaired recovery following MI. Cellular senescence is defined not only by the irreversible loss of division potential but also by the production of a senescence-associated secretory phenotype (SASP). This cocktail of pro-inflammatory cytokines, chemokines, matrix proteases and growth factors can impact on tissue function, inducing fibrosis, extracellular matrix degeneration and driving inflammation. We have therefore begun to test if clearance of senescent cardiomyocytes, using the senolytic compound Navitoclax, has the potential to improve cardiac health and post MI outcomes in aged animals. Following treatment with Navitoclax, but prior to MI, aged mice demonstrated a reduction in senescent cardiomyocytes which was associated increased cardiomyocyte generation, a decline in myocardial hypertrophy and a decrease in fibrosis. Following MI, Navitoclax treated mice displayed a tendency towards improved survival and had a significant improvement in cardiac function when compared to vehicle controls.

We conclude that clearance of senescent cells is a potential therapeutic strategy for the treatment of age related cardiac dysfunction.

\section{ENHANCED ENDOTHELIAL CELL COVERAGE ON GRAPHENE COATED STENTS}

Fatemeh Jafarzadeh*, Daryl McManus, Irina Barbolina, Kostas Kostarelos, Nadim Malik, Cinzia Casiraghi, Cathy M Holt. University of Manchester, 3rd Floor Core Technology Facility, 46 Grafton Street

\subsection{6/heartjnl-2018-BCS.106}

Introduction Coronary artery disease is the leading cause of death worldwide. Stent implantation is performed widely to revascularise stenosed coronary arteries. Restenosis is a significant complication associated with bare metal stents (BMS). Drug eluting stents with adjunct anti-platelet therapy significantly improved restenosis risk but introduced late in stent thrombosis. Therefore, there remains a need for more effective coronary artery stents and the aim of this study is to improve currently available BMSs. Graphene is a $2 \mathrm{D}$ material consisting of a layer of hexagonal structured carbon atoms, exhibiting unique properties, ideal for implantable device coatings: it forms an impermeable membrane, is atomically smooth and has a Daruyhigh surface to volume ratio.

We have coated currently available BMSs with graphenebased materials to enhance re-endothelialisation and hinder the formation of intimal hyperplasia resulting from possible aetiologies such as metal leaching or inflammatory reaction to the metal itself.

Methods Graphene-based bare metal stent coatings were optimised by investigating several coating methods. Human coronary artery endothelial cells (HCAECs) were cultured on control and coated stents. Cells were fixed at day 0 and day 8 post-seeding, followed by Hoechst 33342 and Phalloidin staining for viewing under the fluorescence microscope. Subsequently, HCAEC number per $\mathrm{mm} 2$ area of stent was determined at both day 0 and day 8, and the difference calculated. One-Way ANOVA with multiple comparison was performed and $\mathrm{p}<0.05$ considered as statistically significance. Results are shown as mean \pm SEM. 